\title{
GSplit LBI: Taming the Procedural Bias in Neuroimaging for Disease Prediction
}

\author{
Xinwei Sun ${ }^{1}$, Lingjing $\mathrm{Hu}^{2}(\bowtie)$, Yuan $\mathrm{Yao}^{3}(\bowtie)$, and Yizhou Wang ${ }^{4}$ \\ 1 School of Mathematical Science, Peking University, Beijing, 100871, China \\ ${ }^{2}$ Yanjing Medical College, Capital Medical University, Beijing, 101300, China \\ ${ }^{3}$ Hong Kong University of Science and Technology and Peking University, China \\ ${ }^{4}$ National Engineering Laboratory for Video Technology, Key Laboratory of \\ Machine Perception, School of EECS, Peking University, Beijing, 100871, China
}

\begin{abstract}
In voxel-based neuroimage analysis, lesion features have been the main focus in disease prediction due to their interpretability with respect to the related diseases. However, we observe that there exist another type of features introduced during the preprocessing steps and we call them "Procedural Bias". Besides, such bias can be leveraged to improve classification accuracy. Nevertheless, most existing models suffer from either under-fit without considering procedural bias or poor interpretability without differentiating such bias from lesion ones. In this paper, a novel dual-task algorithm namely GSplit LBI is proposed to resolve this problem. By introducing an augmented variable enforced to be structural sparsity with a variable splitting term, the estimators for prediction and selecting lesion features can be optimized separately and mutually monitored by each other following an iterative scheme. Empirical experiments have been evaluated on the Alzheimer's Disease Neuroimaging Initiative (ADNI) database. The advantage of proposed model is verified by improved stability of selected lesion features and better classification results.
\end{abstract}

Keywords: · Voxel-based Structural Magnetic Resonance Imaging · Procedural Bias · Split Linearized Bregman Iteration · Feature selection

\section{Introduction}

Usually, the first step of voxel-based neuroimage analysis requires preprocessing the $\mathrm{T}_{1}$-weighted image, such as segmentation and registration of grey matter (GM), white matter (WM) and cerebral spinal fluid (CSF). However, some systematic biases due to scanner difference and different population etc., can be introduced in this pipeline [2]. Part of them can be helpful to the discrimination of subjects from normal controls (NC), but may not be directly related to the disease. For example in structural Magnetic Resonance Imaging (sMRI) images of subjects with Alzheimer's Disease (AD), after spatial normalization during simultaneous registration of GM, WM and CSF, the GM voxels surrounding lateral ventricle and subarachnoid space etc. may be mistakenly enlarged caused 
by the enlargement of CSF space in those locations [2] compared to normal template, as shown in Fig. 1. Although these voxels/features are highly correlated with disease, they can't be regarded as lesion features in an interpretable model. In this paper we refer to them as "Procedural Bias", which should be identified but is neglected in the literature. We observe that it can be harnessed in our voxel-based image analysis to improve the prediction of disease.
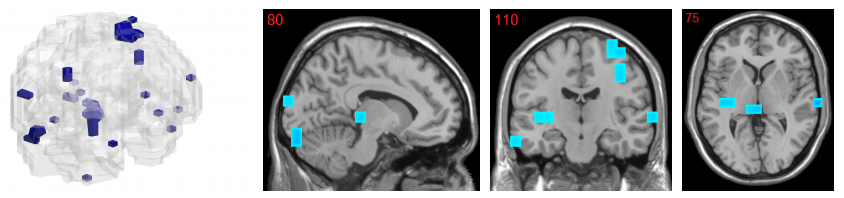

Fig. 1. The overlapped voxels among top 150 negative value voxels in each fold of $\beta_{\text {pre }}$ at the time corresponding to the best average prediction result in the path of GSplit LBI using 10-fold cross-validation. For subjects with AD, they represent enlarged GM voxels surrounding lateral ventricle, subarachnoid space, edge of gyrus, etc.

Together with procedural bias, the lesion features are vital for prediction and lesion regions analysis tasks, which are commonly solved by two types of regularization models. Specifically, one kind of models such as general losses with $l_{2}$ penalty, elastic net [15] and graphnet [5] select strongly correlated features to minimize classification error. However, such models don't differentiate features either introduced by disease or procedural bias and may also introduce redundant features. Hence, the interpretability of such models are poor and the models are prone to over-fit. The other kind of models with sparsity enforcement such as TV- $L_{1}$ (Combination of Total Variation [9] and $L_{1}$ ) and particularly $n^{2}$ GFL [13] enforce strong prior of disease on the parameters of the models introduced in order to capture the lesion features. Although such features are disease-relevant and the selection is stable, the models ignore the inevitable procedural bias, hence, they are losing some prediction power.

To incorporate both tasks of prediction and selection of lesion features, we propose an iterative dual-task algorithm namely Generalized Split LBI (GSplit LBI) which can have better model selection consistency than generalized lasso [11]. Specifically, by the introduction of variable splitting term inspired by Split LBI [6], two estimators are introduced and split apart. One estimator is for prediction and the other is for selecting lesion features, both of which can be pursued separately with a gap control. Following an iterative scheme, they will be mutually monitored by each other: the estimator for selecting lesion features is gradually monitored to pursue stable lesion features; on the other hand, the estimator for prediction is also monitored to exploit both the procedural bias and lesion features to improve prediction. To show the validity of the proposed method, we successfully apply our model to voxel-based sMRI analysis for AD, which is challenging and attracts increasing attention. 


\section{Method}

\subsection{GSplit LBI Algorithm}

Our dataset consists of $N$ samples $\left\{x_{i}, y_{i}\right\}_{1}^{N}$ where $x_{i} \in \mathbb{R}^{p}$ collects the $i^{\text {th }}$ neuroimaging data with $p$ voxels and $y_{i}=\{ \pm 1\}$ indicates the disease status $(-1$ for Alzheimer's disease in this paper). $X \in \mathbb{R}^{N \times p}$ and $y \in \mathbb{R}^{p}$ are concatenations of $\left\{x_{i}\right\}_{i}$ and $\left\{y_{i}\right\}_{i}$. Consider a general linear model to predict the disease status (with the intercept parameter $\beta_{0} \in \mathbb{R}$ ),

$$
\log P\left(y_{i}=1 \mid x_{i}\right)-\log P\left(y_{i}=-1 \mid x_{i}\right)=x_{i}^{T} \beta_{\text {pre }}+\beta_{0} .
$$

A desired estimator $\beta_{\text {pre }} \in \mathbb{R}^{p}$ should not only fit the data by maximizing the loglikelihood in logistic regression, but also satisfy the following types of structural sparsity: (1) the number of voxels involved in the disease prediction is small, so $\beta_{\text {pre }}$ is sparse; (2) the voxel activities should be geometrically clustered or 3Dsmooth, suggesting a TV-type sparsity on $D_{G} \beta_{\text {pre }}$ where $D_{G}$ is a graph difference operator $^{5}$; (3) the degenerate GM voxels in $\mathrm{AD}$ are captured by nonnegative component in $\beta_{\text {pre }}$. However, the existing procedural bias may violate these $a$ priori sparsity properties, esp. the third one, yet increase the prediction power.

To overcome this issue, we adopt a variable splitting idea in [6] by introducing an auxiliary variable $\gamma \in \mathbb{R}^{|V|+|E|}$ to achieve these sparsity requirements separately, while controlling the gap from $D \beta_{\text {pre }}$ with penalty $S_{\rho}\left(\beta_{\text {pre }}, \gamma\right):=$ $\left\|D \beta_{\text {pre }}-\gamma\right\|_{2}^{2}:=\left\|\beta_{\text {pre }}-\gamma_{V}\right\|_{2}^{2}+\left\|\rho D_{G} \beta_{\text {pre }}-\gamma_{G}\right\|_{2}^{2}$ with $\gamma=\left[\gamma_{V}^{T} \gamma_{G}^{T}\right]^{T}$ and $D=\left[\begin{array}{ll}I & \rho \\ D_{G}^{T}\end{array}\right]^{T}$. Here $\rho$ controls the trade-off between different types of sparsity. Our purpose is thus of two-folds: (1) use $\beta_{\text {pre }}$ for prediction; (2) enforce sparsity on $\gamma$. Such a dual-task scheme can be illustrated by Fig. 6 .

To implement it, we generalize the Split Linearized Bregman Iteration (Split LBI) algorithm in [6] to our setting with generalized linear models (GLM) and the three types of structural sparsity above, hence called Generalized Split LBI (or GSplit LBI). Algorithm 1 describes the procedure with a new loss:

$$
\ell\left(\beta_{0}, \beta_{\text {pre }}, \gamma ;\left\{x_{i}, y_{i}\right\}_{1}^{N}, \nu\right):=\ell\left(\beta_{0}, \beta_{\text {pre }} ;\left\{x_{i}, y_{i}\right\}_{1}^{N}\right)+\frac{1}{2 \nu} S_{\rho}\left(\beta_{\text {pre }}, \gamma\right),
$$

where $\ell\left(\beta_{\text {pre }} ;\left\{x_{i}, y_{i}\right\}_{1}^{N}\right)$ is the negative log-likelihood function for GLM and $\nu>$ 0 tunes the strength of gap control. The algorithm returns a sequence of estimates as a regularization path, $\left\{\beta_{0}^{k}, \beta_{\text {pre }}^{k}, \gamma^{k}, \beta_{l e s}^{k}\right\}_{k \geq 0}$. In particular, $\gamma^{k}$ shows a variety of sparsity levels and $\beta_{p r e}^{k}$ is generically dense with different prediction powers. The projection of $\beta_{p r e}^{k}$ onto the subspace with the same support of $\gamma^{k}$ gives estimate $\beta_{\text {les }}^{k}$, satisfying those a priori sparsity properties (sparse, 3D-smooth, nonnegative) and hence being regarded as the interpretable lesion features for AD. The remainder of this projection is heavily influenced by procedural bias; in

\footnotetext{
${ }^{5}$ Here $D_{G}: \mathbb{R}^{V} \rightarrow \mathbb{R}^{E}$ denotes a graph difference operator on $G=(V, E)$, where $V$ is the node set of voxels, $E$ is the edge set of voxel pairs in neighbour (e.g. 3-by-3-by-3), such that $D_{G}(\beta)(i, j):=\beta(i)-\beta(j)$.
} 


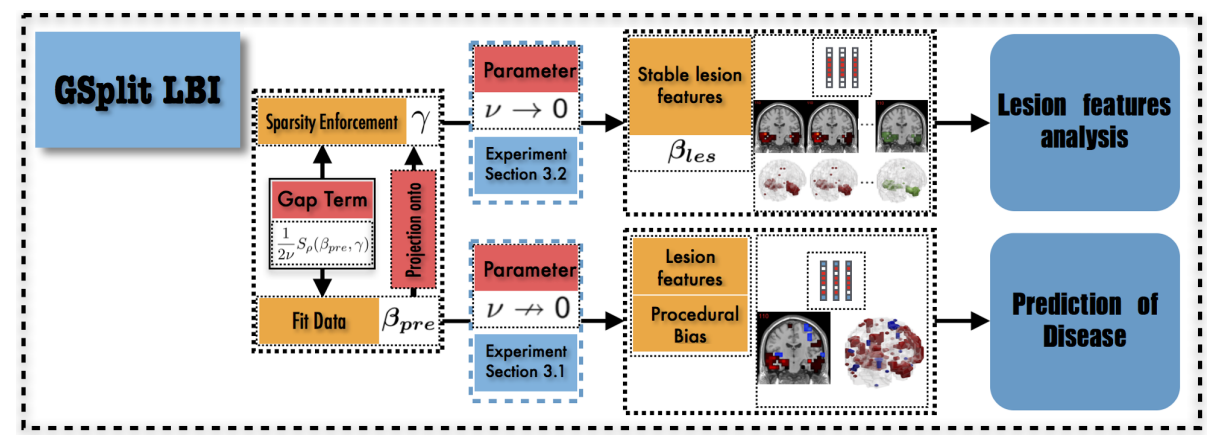

Fig. 2. Illustration of GSplit LBI. The gap between $\beta_{\text {pre }}$ for fitting data and $\gamma$ for sparsity is controlled by $S_{\rho}\left(\beta_{\text {pre }}, \gamma\right)$. The estimate $\beta_{\text {les }}$, as a projection of $\beta_{\text {pre }}$ on support set of $\gamma$, can be used for stable lesion features analysis when $\nu \rightarrow 0$ (Section 3.2). When $\nu \nrightarrow 0$ (Section 3.1) with appropriately large value, $\beta_{\text {pre }}$ can be used for prediction by capturing both lesion features and procedural bias.

this paper the non-zero elements in $\beta_{p r e}^{k}$ which are negative ( -1 denotes disease label) with comparably large magnitude are identified as procedural bias, while others with tiny values can be treated as nuisance or weak features. In summary, $\beta_{\text {les }}$ only selects lesion features; while $\beta_{\text {pre }}$ also captures additional procedural bias. Hence, such two kinds of features can be differentiated, as illustrated in Fig. 6.

\subsection{Setting the Parameters}

A stopping time at $t^{k}$ (line 10) is the regularization parameter, which can be determined via cross-validation to minimize the prediction error [7]. Parameter $\rho$ is a tradeoff between geometric clustering and voxel sparsity. Parameter $\kappa, \alpha$ is damping factor and step size, which should satisfy $\kappa \alpha \leq \nu / \kappa\left(1+\nu \Lambda_{H}+\Lambda_{D}^{2}\right)$ to ensure the stability of iterations. Here $\Lambda_{(\cdot)}$ denotes the largest singular value of a matrix and $H$ denotes the Hessian matrix of $\ell\left(\beta_{0}, \beta_{\text {pre }} ;\left\{x_{i}, y_{i}\right\}_{1}^{N}\right)$.

Parameter $\nu$ balances the prediction task and sparsity enforcement in feature selection. In this paper, it is task-dependent, as shown in Fig. 6. For prediction of disease, $\beta_{\text {pre }}$ with appropriately larger value of $\nu$ may increase the prediction power by harnessing both lesion features and procedural bias. For lesion features analysis, $\beta_{\text {les }}$ with a small value of $\nu$ is helpful to enhance stability of feature selection. For details please refer to supplementary information.

\section{Experimental Results}

We apply our model to AD/NC classification (namely ADNC) and MCI (Mild Cognitive Impairment)/NC (namely MCINC) classification, which are two fundamental challenges in diagnosis of $\mathrm{AD}$. The data are obtained from $\mathrm{ADNI}^{6}$

\footnotetext{
$\overline{{ }^{6} \text { http://adni.loni.ucla.edu }}$
} 


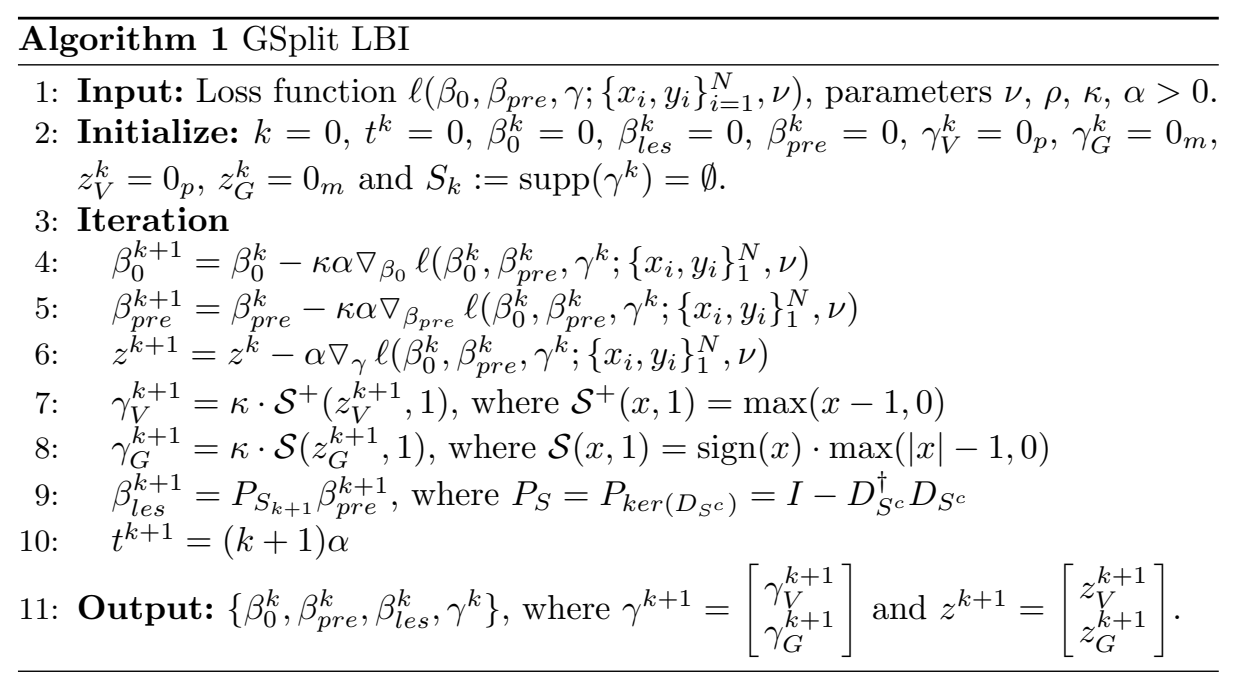

database, which is split into $1.5 \mathrm{~T}$ and 3.0T (namely 15 and 30) MRI scan magnetic field strength datasets. The 15 dataset contains 64 AD, 208 MCI and 90 NC; while the 30 dataset contains $66 \mathrm{AD}$ and 110 NC. DARTEL VBM pipeline [1] is then implemented to preprocess the data. Finally, the input features consist of $2,5278 \times 8 \times 8 \mathrm{~mm}^{3}$ size voxels with average values in GM population template greater than 0.1. Experiments are designed on 15ADNC, 30ADNC and 15MCINC tasks.

\subsection{Prediction and Path Analysis}

10 -fold cross-validation is adopted for classification evaluation. Under exactly the same experimental setup, comparison is made between GSplit LBI and other classifiers: SVM, MLDA (univariate model via t-test + LDA) [3], Graphnet [5], Lasso [10], Elastic Net, TV $+\mathrm{L}_{1}$ and $n^{2}$ GFL. For each model, optimal parameters are determined by grid-search. For GSplit LBI, $\rho$ is chosen from $\{1,2, \ldots, 10\}$, $\kappa$ is set to $10 ; \alpha=\nu / \kappa\left(1+\nu \Lambda_{X}^{2}+\Lambda_{D}^{2}\right)^{7}$; specifically, $\nu$ is set to 0.2 (corresponding to $\nu \nrightarrow 0$ in Fig. 6$)^{8}$. The regularization coefficient $\lambda$ is ranged in $\left\{0,0.05,0.1, \ldots, 0.95,1,10,10^{2}\right\}$ for lasso 9 and $2^{\{-20,-19, \ldots, 0, \ldots, 20\}}$ for SVM. For other models, parameters are optimized from $\lambda:\left\{0.05,0.1, \ldots, 0.95,1,10,10^{2}\right\}$ and $\rho:\{0.5,1, . ., 10\}$ (in addition, the mixture parameter $\alpha:\{0,0.05, \ldots, 0.95\}$ for Elastic Net).

The best accuracy in the path of GSplit LBI and counterpart are reported. Table 1 shows that $\beta_{\text {pre }}$ of our model outperforms that of others in all cases. Note that although our accuracies may not be superior to models with multi-modality data [8], they are the state-of-the-art results for only sMRI modality.

\footnotetext{
${ }^{7}$ For logit model, $\alpha<\nu / \kappa\left(1+\nu \Lambda_{H}^{2}+\nu \Lambda_{X}^{2}\right)$ since $\Lambda_{X}>\Lambda_{H}$.

${ }^{8}$ In this experiment, comparable prediction result will be given for $\nu \in(0.1,10)$.

${ }^{9} 0$ corresponds to logistic regression model.
} 
Table 1. Comparison of GSplit LBI with other models

\begin{tabular}{c|c|c|c|c|c|c|c|c}
\hline & MLDA & SVM & Lasso & Graphnet & Elastic Net & TV $+l_{1}$ & $n^{2}$ GFL & GSplit LBI $\left(\beta_{\text {pre }}\right)$ \\
\hline 15ADNC & $85.06 \%$ & $83.12 \%$ & $87.01 \%$ & $86.36 \%$ & $88.31 \%$ & $83.77 \%$ & $86.36 \%$ & $\mathbf{8 8 . 9 6 \%}$ \\
\hline 30ADNC & $86.93 \%$ & $87.50 \%$ & $87.50 \%$ & $88.64 \%$ & $89.20 \%$ & $87.50 \%$ & $87.50 \%$ & $\mathbf{9 0 . 9 1 \%}$ \\
\hline 15MCINC & $61.41 \%$ & $70.13 \%$ & $69.80 \%$ & $72.15 \%$ & $70.13 \%$ & $73.83 \%$ & $69.80 \%$ & $\mathbf{7 5 . 1 7 \%}$ \\
\hline
\end{tabular}
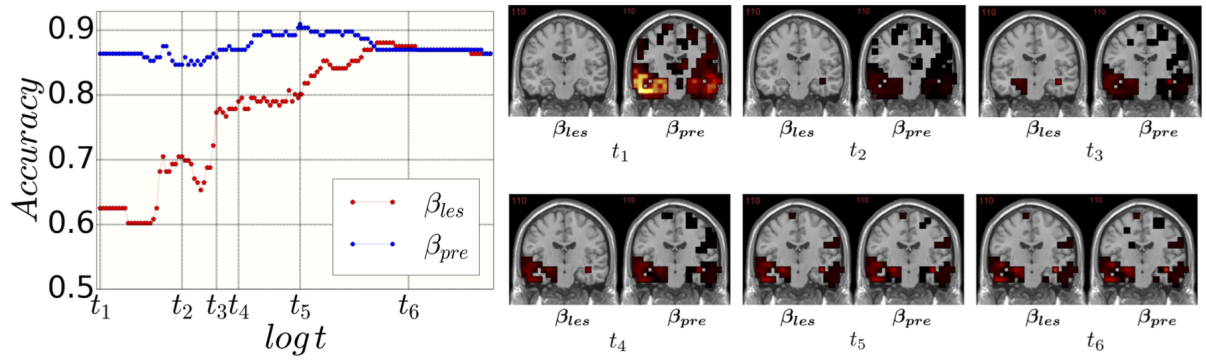

Fig. 3. Left image: Accuracy of $\left(\beta_{\text {pre }}, \beta_{\text {les }}\right)$ vs $\log t(t$ : regularization parameter). Right image: Six 2 -d brain slice images of selected degenerative voxels of $\beta_{\text {les }}$ and $\beta_{\text {pre }}$ are sorted orderly at $\left\{t_{1}, \ldots t_{6}\right\}$. As $t$ grows, $\beta_{\text {pre }}$ and $\beta_{\text {les }}$ identify similar lesion features.

The process of feature selection combined with prediction accuracy can be analyzed together along the path. The result of 30ADNC is used as an illustration in Fig. 3. We can see that $\beta_{\text {pre }}$ (blue curve) outperforms $\beta_{\text {les }}$ (red curve) in the whole path for additional procedural bias captured by $\beta_{\text {pre }}$. Specifically, at $\beta_{\text {pre }}$ 's highest accuracy $\left(t_{5}\right)$, there is a more than $8 \%$ increase in prediction accuracy by $\beta_{\text {pre }}$. Early stopping regularization at $t_{5}$ is desired, as $\beta_{\text {pre }}$ converges to $\beta_{\text {les }}$ in prediction accuracy with overfitting when $t$ grows. Recall that positive (negative) features represent degenerate (enlarged) voxels. In each fold of $\beta_{\text {pre }}$ at $t_{5}$, the commonly selected voxels among top 150 negative (enlargement) voxels are identified as procedural bias shown in Fig. 1, where most of these GM voxels are enlarged and located near lateral ventricle or subarachnoid space etc., possibly due to enlargement of CSF space in those locations that are different from the lesion features.

\subsection{Lesion Features Analysis}

To quantitatively evaluate the stability of selected lesion features, multi-set Dice Coefficient $(\mathrm{mDC})^{10}[4,13]$ is applied as a measurement. The 30ADNC task is again applied as an example, the $\mathrm{mDC}$ is computed for $\beta_{\text {les }}$ which achieves highest accuracy by 10 -fold cross-validation. As shown from Table 2 , when $\nu=$ 0.0002 (corresponding to $\nu \rightarrow 0$ in Fig. 6), the $\beta_{\text {les }}$ of our model can obtain more stable lesion feature selection results than other models with comparable

${ }^{10} \operatorname{In}[13], m D C:=\frac{10\left|\cap_{k=1}^{10} S(k)\right|}{\sum_{k=1}^{10}|S(k)|}$ where $S(k)$ denotes the support set of $\beta_{\text {les }}$ in k-th fold. 
prediction power. Besides, the average number of selected features (line 3 in Table 2) are also recorded. Note that although elastic net is of slightly higher accuracy than $\beta_{\text {les }}$, it selects much more features than necessary.

Table 2. mDC comparison between GSplit LBI and other models

\begin{tabular}{c|c|c|c|c|c|c}
\hline & Lasso & Elastic Net & Graphnet & TV $+l_{1}$ & $n^{2}$ GFL & GSplit LBI $\left(\beta_{\text {les }}\right)$ \\
\hline Accuracy & $87.50 \%$ & $89.20 \%$ & $88.64 \%$ & $87.50 \%$ & $87.50 \%$ & $88.64 \%$ \\
\hline mDC & 0.1992 & 0.5631 & 0.6005 & 0.5824 & 0.5362 & $\mathbf{0 . 7 8 0 5}$ \\
\hline$\sum_{k=1}^{10}|S(k)| / 10$ & 50.2 & 777.8 & 832.6 & 712.6 & 443.9 & 129.4 \\
\hline
\end{tabular}

For the meaningfulness of selected lesion features, they are shown in Fig. 4 (a)-(c), located in hippocampus, parahippocampal gyrus and medial temporal lobe etc., which are believed to be early damaged regions for $\mathrm{AD}$ patients.

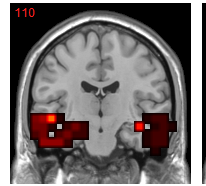

(a) fold 2

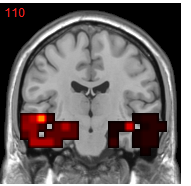

(b) fold 10

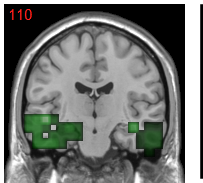

(c) overlap

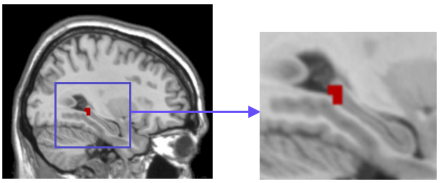

(d) coarse-to-fine

Fig. 4. (a)-(c): Stability of selected lesion features of $\beta_{\text {les }}$ shown in 2-d 110 slice brain images when $\nu=0.0002$. (a)-(b): Results of fold 2 and fold 10. (c): The overlapped features in 10 folds. (d): The 2 -d slice brain image of selected voxels with $2 \times 2 \times 2$ $\mathrm{mm}^{3}$ using coarse-to-fine approach.

To further investigate the locus of lesion features, we conduct a coarse-tofine experiment. Specifically, we project the selected overlapped voxels of $8 \times$ $8 \times 8 \mathrm{~mm}^{3}$ size (shown in Fig. 4 (c)) onto MRI image with more finer scale voxels, i.e. in size of $2 \times 2 \times 2 \mathrm{~mm}^{3}$. Totally 4,895 voxels are served as input features after projection. Again, the GSplit LBI is implemented using 10-fold cross-validation. The prediction accuracy of $\beta_{p r e}$ is $90.34 \%$ and on average 446.6 voxels are selected by $\beta_{\text {les }}$. As desired, these voxels belong to parts of lesion regions, such as those located in hippocampal tail, as shown in Fig. 4 (d).

\section{Conclusions}

In this paper, a novel iterative dual task algorithm is proposed to incorporate both disease prediction and lesion feature selection in neuroimage analysis. 
With variable splitting term, the estimators for prediction and selecting lesion features can be separately pursued and mutually monitored under a gap control. The gap here is dominated by procedural bias, some specific features crucial for prediction yet ignored in a priori disease knowledge. With experimental studies conducted on 15ADNC, 30ADNC and 15MCINC tasks, we have shown that the leverage of procedural bias can lead to significant improvements in both prediction and model interpretability. In future works, we shall extend our model to other neuroimaging applications including multi-modality data.

Acknowledgements. This work was supported in part by 973-2015CB351800, 2015CB85600, 2012CB825501, NSFC-61625201, 61370004, 11421110001 and Scientific Research Common Program of Beijing Municipal Commission of Education (No. KM201610025013).

\section{References}

1. Ashburner, J.: A fast diffeomorphic image registration algorithm. Neuroimage 38(1), 95-113 (2007)

2. Ashburner, J., Friston, K.J.: Why voxel-based morphometry should be used. Neuroimage $14(6), 1238-1243$ (2001)

3. Dai, Z., Yan, C., Wang, Z., Wang, J., Xia, M., Li, K., He, Y.: Discriminative analysis of early alzheimer's disease using multi-modal imaging and multi-level characterization with multi-classifier. Neuroimage 59(3), 2187-2195 (2012)

4. Dice, L.R.: Measures of the amount of ecologic association between species. Ecology 26(3), 297-302 (1945)

5. Grosenick, L., Klingenberg, B., Katovich, K., Knutson, B., Taylor, J.E.: Interpretable whole-brain prediction analysis with graphnet. Neuroimage 72, 304-321 (2013)

6. Huang, C., Sun, X., Xiong, J., Yao, Y.: Split lbi: An iterative regularization path with structural sparsity. advances in neural information processing systems. Advances In Neural Information Processing Systems pp. 3369-3377 (2016)

7. Osher, S., Ruan, F., Xiong, J., Yao, Y., Yin, W.: Sparse recovery via differential inclusions. Applied and Computational Harmonic Analysis (2016)

8. Peng, J., An, L., Zhu, X., Jin, Y., Shen, D.: Structured sparse kernel learning for imaging genetics based alzheimer's disease diagnosis. International Conference on Medical Image Computing and Computer-Assisted Intervention pp. 70-78 (2016)

9. Rudin, L.I., Osher, S., Fatemi, E.: Nonlinear total variation based noise removal algorithms. Physica D: Nonlinear Phenomena 60(1-4), 259-268 (1992)

10. Tibshirani, R.: Regression shrinkage and selection via the lasso. Journal of the Royal Statistical Society. Series B (Methodological) 58, 267-288 (1996)

11. Tibshirani, R.J., Taylor, J.E., Candes, E.J., Hastie, T.: The solution path of the generalized lasso. The Annals of Statistic 39(3), 1335-1371 (2011)

12. Vaiter, S., Peyré, G., Dossal, C., Fadili, J.: Robust sparse analysis regularization. IEEE Transactions on Information Theory 59(4), 2001-2016 (2013)

13. Xin, B., Hu, L., Wang, Y., Gao, W.: Stable feature selection from brain smri. AAAI pp. 1910-1916 (2014)

14. Yin, W., Osher, S., Darbon, J., Goldfarb, D.: Bregman iterative algorithms for compressed sensing and related problems. SIAM Journal on Imaging Sciences 1(1), $143-168$ (2008) 
15. Zou, H., Hastie, T.: Regularization and variable selection via the elastic net. Journal of the Royal Statistical Society: Series B (Statistical Methodology) 67(2), 301$320(2005)$ 


\section{Supplementary Information}

\section{A Notation}

For matrix $A, A_{J}$ represents the submatrix of $A$ indexed by $J . A^{\dagger}$ denotes the Moore-Penrose pseudoinverse of $A$. Suppose $A \in R^{n \times n},\|A\|_{\Sigma}:=\operatorname{trace}(A)=$ $\sum_{i=1}^{n} A_{i, i}$. Besides, $\tilde{\beta}$ and $\beta$ are used to represent $\beta_{\text {les }}$ and $\beta_{\text {pre }}$ respectively in what follows.

\section{B Model selection consistency}

Consider recovery from generalized linear model(GLM) of $\beta^{\star} \in R^{p}$ which satisfies structural sparsity after linearly transformed by $D \in R^{m \times p}$ :

$$
\begin{gathered}
P\left(y \mid x, \beta^{\star}\right) \propto \exp \left(\frac{x^{T} \beta^{\star} \cdot y-\psi\left(x^{T} \beta^{\star}\right)}{d(\sigma)}\right) \\
\text { s.t. } \quad \gamma^{\star}=D \beta^{\star}\left(S:=\operatorname{supp}\left(\gamma^{\star}\right), s=|S|, s<<m\right)
\end{gathered}
$$

where $\psi: R \rightarrow R$ is link function and $d(\sigma)$ is known parameter related to the variance of distribution.

Under linear model with $\psi(t)=t^{2}$ and $d(\sigma)=\sigma^{2}$ in B.1, our model GSplit LBI degenerates to Split LBI [6]. Recently, it's proved in [6] that the Split LBI may achieve model selection consistency under weaker conditions than generalized lasso $[11,12]$ if $\nu$ is large enough. We claim that this property can also be shared by logit model.

To understand why Gsplit LBI can achieve better model selection consistency, note that the variable splitting term projects solution vector $\beta$ into higher dimensional space $(\beta, \gamma)$ with $\beta$ fitting data and $\gamma$ being structural sparse. This will make it easier for the subspace of $\gamma_{S^{c}}$ to decorrelate with the subspace of $\left(\beta, \gamma_{S}\right)$, especially when $\nu$ increases, which sheds light on better performance of Split LBI to recover true signal set $S$. What's more important, the property may also be shared by logit model when $y=\{ \pm 1\}, d(\sigma)=1$ and $\psi(t)=\log (1+\exp (t))$. Concretely speaking, we use $\theta_{S^{c},(\beta, S)}(\nu)$ to denote the angle between subspace of $\gamma_{S^{c}}$ and that of $\left(\beta, \gamma_{S}\right)$, the definition of which is:

$\theta_{S^{c},(\beta, S)}(\nu):=\arccos \left(\frac{\left\|P_{A_{(\beta, S)}} A_{S^{c}}\right\|_{F}}{\left\|A_{S^{c}}\right\|_{F}}\right)=\arccos \left(\sqrt{\frac{\left\|H_{S^{c},(\beta, S)} H_{(\beta, S),(\beta, S)}^{\dagger} H_{(\beta, S), S^{c}}\right\|_{\Sigma}}{\left\|H_{S^{c}, S^{c}}\right\|_{\Sigma}}}\right)$

Where $A:=\left[A_{(\beta, S)} A_{S^{c}}\right]$ and $H:=\nabla_{\beta, \gamma}^{2} l(\beta, \gamma)=A^{T} A=\left[\begin{array}{cc}H_{(\beta, S),(\beta, S)} & H_{(\beta, S), S^{c}} \\ H_{S^{c},(\beta, S)} & H_{S^{c}, S^{c}}\end{array}\right]$.

Remark 1. For linear model, $A=\nabla_{\beta, \gamma} l(\beta, \gamma)$ with

$$
A_{(\beta, S)}=\left[\begin{array}{cc}
X & 0_{n \times s} \\
-D_{S} / \sqrt{\nu} & I_{(S, S)} / \sqrt{\nu} \\
-D_{S^{c}} / \sqrt{\nu} & 0_{(p-s) \times s}
\end{array}\right] \quad A_{S^{c}}=\left[\begin{array}{c}
0_{n \times(p-s)} \\
0_{s \times(p-s)} \\
I_{\left(S^{c}, S^{c}\right)} / \sqrt{\nu}
\end{array}\right]
$$


There is no explicit definition for $A$ for logit model, however $\theta_{S^{c},(\beta, S)}(\nu)$ can be computed through Hessian matrix $H$ in equation B.3.

We claim that $\theta_{S^{c},(\beta, S)}(\nu)$ will increase as $\nu$ becomes larger under some conditions. See theorem 1 for details.

Theorem 1. Under linear model and logit model, $\lim _{\nu \rightarrow+\infty} \theta_{S^{c},(\beta, S)}(\nu)=90^{\circ}$ if and only if $\operatorname{Im}\left(D_{S^{c}}^{T}\right) \subseteq \operatorname{Im}\left(X^{T}\right)$.

Remark 2. In [6], it's been proved that the necessary condition for sign-consistency is $\operatorname{IRR}(\nu)<1$. For uniqueness of model, we also assume that $\operatorname{ker}(X) \cap \operatorname{ker}\left(D_{S^{c}}\right)$ $\subseteq \operatorname{ker}\left(D_{S}\right)$. Combined with $\operatorname{Im}\left(D_{S^{c}}^{T}\right) \subseteq \operatorname{Im}\left(X^{T}\right) \Longleftrightarrow \operatorname{ker}(X) \subseteq \operatorname{ker}\left(D_{S^{c}}\right)$, we have that $\operatorname{ker}(X) \subseteq \operatorname{ker}\left(D_{S}\right)$, which is the sufficient and necessary condition for the hold of $\lim _{\nu \rightarrow \infty} \operatorname{IRR}(\nu) \rightarrow 0$. Hence, this is another way to understand why GSplit LBI can achieve better model selection consistency.

Proof. We firstly prove the case under linear model. Denoted $A:=\nu X^{\star} X+D^{T} D$ where $X \in R^{n \times p}$ and $X^{\star}=X / n$. Note that:

$$
H_{(\beta, S),(\beta, S)}=Q L Q^{T}, \quad H_{S^{c},(\beta, S)}=\left[D_{S^{c}} / \nu 0\right]
$$

where:

$$
Q=\left[\begin{array}{cc}
I_{p} & 0 \\
-D_{S} A^{\dagger} & I_{s}
\end{array}\right], \quad L=\left[\begin{array}{cc}
A / \nu & 0 \\
0 & \left(I_{s}-D_{S} A^{\dagger} D_{S}^{T}\right) / \nu
\end{array}\right]
$$

Then we have:

$$
H_{S^{c},(\beta, S)} H_{(\beta, S),(\beta, S)}^{\dagger} H_{(\beta, S), S^{c}}=H_{S^{c},(\beta, S)} Q L^{\dagger} Q^{T} H_{(\beta, S), S^{c}}=\frac{1}{\nu} D_{S^{c}} A^{\dagger} D_{S^{c}}^{T}
$$

Substituting equation B.5 into the second equation of B.2, we have:

$$
\cos ^{2}\left(\theta_{S^{c},(\beta, S)}(\nu)\right)=\frac{\left\|D_{S^{c}} A^{\dagger} D_{S^{c}}\right\|_{\Sigma}}{\left\|H_{S^{c}, S^{c}}\right\|_{\Sigma}}=\frac{\left\|D_{S^{c}} A^{\dagger} D_{S^{c}}\right\|_{\Sigma}}{m-s}
$$

Denote $e_{i} \in R^{m-s}$ as the vector with the $i^{\text {th }}$ element being 1 and left being 0 . Then equation B.6 is equivalent to:

$$
\cos ^{2}\left(\theta_{S^{c},(\beta, S)}(\nu)\right)(m-s)=\Sigma_{i=1}^{p} d_{i}^{T} A^{\dagger} d_{i}
$$

where $d_{i}:=D_{S^{c}}^{T} e_{i}$. Suppose the compact singular value decomposition of $X / \sqrt{n}:=$ $U \Lambda V^{T}$, and $(V, \tilde{V})$ be an orthogonal square matrix. Suppose the compact singular value decomposition of $D \tilde{V}:=U_{1} \Lambda_{1} V_{1}^{T}$. If $\operatorname{Im}\left(D_{S^{c}}^{T}\right) \subseteq \operatorname{Im}\left(X^{T}\right)$, then $\exists f_{i}$, such that $d_{i}=V f_{i}$, hence,

$$
\begin{aligned}
d_{i}^{T}\left(\nu X^{\star} X+D^{T} D\right)^{\dagger} d_{i} & =d_{i}^{T}(V \tilde{V})\left(\left(\begin{array}{c}
V^{T} \\
\tilde{V}^{T}
\end{array}\right)\left(\nu X^{\star} X+D^{T} D\right)(V \tilde{V})\right)^{\dagger}\left(\begin{array}{c}
V^{T} \\
\tilde{V}^{T}
\end{array}\right) d_{i} \\
& =f_{i}^{T}\left(\nu \Lambda^{2}+V^{T} D^{T} D V\right)^{-1} f_{i} \rightarrow 0, \text { as } \nu \rightarrow \infty
\end{aligned}
$$


Combined with equation B.7, it's then easy to obtain that $\cos ^{2}\left(\theta_{S^{c},(\beta, S)}(\nu)\right) \rightarrow 0$ as $\nu \rightarrow+\infty$. On the contrary, if $\exists a$ such that $D_{S^{c}}^{T} a \notin \operatorname{Im}\left(X^{T}\right)$, then there $\exists i^{\star}$ such that $d_{i^{\star}} \notin \operatorname{Im}\left(X^{T}\right)$. This means that for $d_{i^{\star}}$, there $\exists f_{1, i^{\star}}, f_{2, i^{\star}} \neq 0$ such that $d_{i^{\star}}=V f_{1, i^{\star}}+\tilde{V} f_{2, i^{\star}}$. Then we have

$$
d_{i^{\star}}^{T}\left(\nu X^{\star} X+D^{T} D\right)^{\dagger} d_{i^{\star}} \geq f_{2, i^{\star}}^{T}\left(\tilde{V}^{T} D^{T} D \tilde{V}\right)^{\dagger} f_{2, i^{\star}}=f_{2, i^{\star}}^{T} V_{1} \Lambda_{1}^{-2} V_{1}^{T} f_{2, i^{\star}}
$$

does not equal to $0 \Longleftrightarrow f_{2, i^{\star}}^{T} V_{1} \Lambda_{1}^{2} V_{1}^{T} f_{2, i^{\star}}=f_{2, i^{\star}}^{T} \tilde{V}^{T} D^{T} D \tilde{V} f_{2, i^{\star}} \neq 0$. Since

$$
f_{2, i^{\star}}^{T} \tilde{V}^{T} D^{T} D \tilde{V} f_{2, i^{\star}} \geq f_{2, i^{\star}}^{T} \tilde{V}^{T} d_{i^{\star}} d_{i^{\star}}^{T} f_{2, i^{\star}}=\left(f_{2, i^{\star}}^{T} f_{2, i^{\star}}\right)^{2}>0
$$

From equation B.7, we can obtain that:

$$
\cos ^{2}\left(\theta_{S^{c},(\beta, S)}(\nu)\right)(m-s) \geq d_{i^{\star}}^{T} A^{\dagger} d_{i^{\star}} \neq 0
$$

which means the $\theta_{S^{c},(\beta, S)}(\nu) \rightarrow 0$ does not hold when $\nu \rightarrow+\infty$. The proof is then completed under linear model. Under logit model, the definition of $A$ is modified to $A:=\nu X^{\star} W\left(\left\{x_{i}, \beta\right\}_{i=1}^{p}\right) X+D^{T} D$ where $W\left(\left\{x_{i}, \beta\right\}_{i=1}^{p}\right)$ is a diagonal matrix with each diagonal element equals to $\frac{\exp \left(x_{i}^{T} \beta\right)}{\left(1+\exp \left(x_{i}^{T} \beta\right)\right)^{2}}$, the left proof is almost the same with that of linear model.

An simulation experiment is conducted to illustrate this idea. In more detail, $n=100$ and $p=80, D=I$ and $X \in R^{n \times p}$ and $X_{i, j} \sim N(0,1) . \beta_{i}^{\star}=2$ for $1 \leq i \leq 4, \beta_{i}^{\star}=-2$ for $5 \leq i \leq 8$ and 0 otherwise, $y$ is generated by both linear model $y=X \beta^{\star}+\epsilon$ with $\epsilon \sim N(0,1)$ and logit model given $X$ and $\beta^{\star}$. We simulated for 100 times and average $\theta_{S^{c},(\beta, S)}(\nu)$ is then computed, which is shown in the left image in figure 5 . We can see that $\theta_{S^{c},(\beta, S)}(\nu)$ increases when $\nu$ becomes larger, as illustrated in right image in figure 5 , and converges to $90^{\circ}$ when $\nu \rightarrow+\infty$.

The average AUC and estimation of $\beta^{\star}$ of Gsplit LBI with different $\nu$ compared with those of genlasso are also computed. Table B shows better AUC with the increase of $\nu$ before $\nu=100$. As we can see from the algorithm in the paper that $\tilde{\beta}$ is the projection of $\beta$ onto the support set of $\gamma$. Hence it is equivalent to say that better model selection of $\tilde{\beta}$ can be achieved as $\nu$ increases.

However, the excessively large value of $\nu$ will lower the signal-to-noise ratio, which is also crucial for model selection consistency and prediction estimation. It's shown in [6] that $\nu$ determines the trade-off between model selection consistency and estimation of $\beta^{\star}$. Also, the irrepresentable condition(IRR) can be satisfied as long as $\nu$ is large enough. If $\nu$ continuously increase, it will deteriorate the estimation of $\beta^{\star}$, prediction estimation and even AUC. In our experiment the same phenomena can be observed, i.e. the estimation of $\tilde{\beta}$ and $\beta$ get worse if $\nu$ increases from 10 and 100, respectively; when $\nu=100$, AUC even decreases.

\section{Relationship between $\boldsymbol{\beta}$ and $\tilde{\boldsymbol{\beta}}$}

The estimate $\tilde{\beta}$, as a projection of $\beta$ onto the subspace of $\gamma$, can select features that satisfy structural sparsity. Following the Linearized Bregman Iteration [14], 

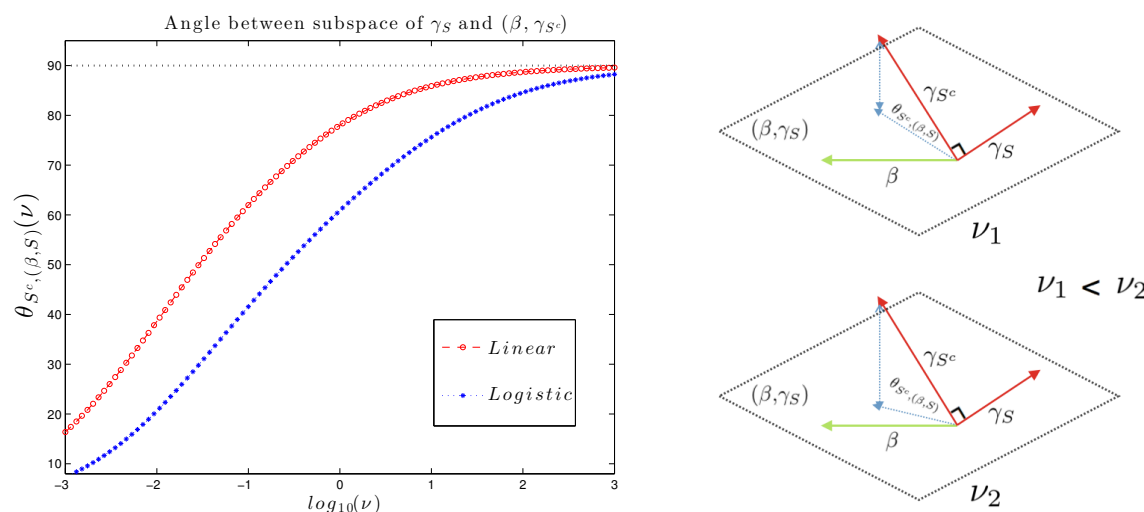

Fig. 5. Left image: The $\theta_{S^{c},(\beta, S)}(\nu)$ curve of logit model and linear model. Right image: Illustration of $\theta_{S^{c},(\beta, S)}(\nu)$ and it monotonically increase v.s. $\nu$.

Table 3. Comparison between Gsplit LBI with different $\nu$ and genlasso in terms of AUC, $\left\|\tilde{\beta}-\beta^{\star}\right\|_{2},\left\|\beta-\beta^{\star}\right\|_{2},\left\|X \tilde{\beta}-X \beta^{\star}\right\|_{2}$ and $\left\|X \beta-X \beta^{\star}\right\|_{2}$.

\begin{tabular}{|c|c|c|c|c|c|c|c|}
\hline Model & \multicolumn{6}{|c|}{ Gsplit LBI } & genlasso \\
\hline$\nu$ & 0.02 & 0.1 & 1 & 5 & 10 & 100 & - \\
\hline$\overline{\mathrm{AUC}}$ & 0.9531 & 0.98194 & 0.98514 & 0.98791 & \begin{tabular}{|l|}
0.98792 \\
\end{tabular} & 0.98590 & 0.97915 \\
\hline $\mid \tilde{\beta}-\beta^{\star} \|_{2}$ & 4.9079 & 4.9015 & 4.8513 & 4.8495 & 4.8473 & 5.3578 & - \\
\hline $\mid \beta-\beta^{\star} \|_{2}$ & 3.9187 & 3.8993 & 3.7619 & 3.6814 & \begin{tabular}{|l|}
3.7129 \\
\end{tabular} & 5.1540 & 4.9113 \\
\hline$X \tilde{\beta}-X \beta^{\star} \|_{2}$ & 47.0224 & 46.9625 & 46.6784 & 46.9158 & 47.0098 & 52.2737 & - \\
\hline$X \beta-X \beta^{\star} \|_{2}$ & 37.3496 & 37.0962 & 35.4987 & 34.6423 & 34.8514 & 49.61020 & 50.6408 \\
\hline
\end{tabular}

$\beta$ and $\tilde{\beta}$ will be more similar on features selected by $\tilde{\beta}$. In more detail, note that when $t=0, \tilde{\beta}(t)=0$ and $\beta(t)$ is the graph laplacian regularizer with penalty factor $\frac{1}{2 \nu}$. As $t$ progresses, the gap between $\beta(t)$ and $\tilde{\beta}(t)$ will decrease in terms of $\|\beta(t)-\tilde{\beta}(t)\|_{2}$ for every $\nu$, as shown in figure 6 .
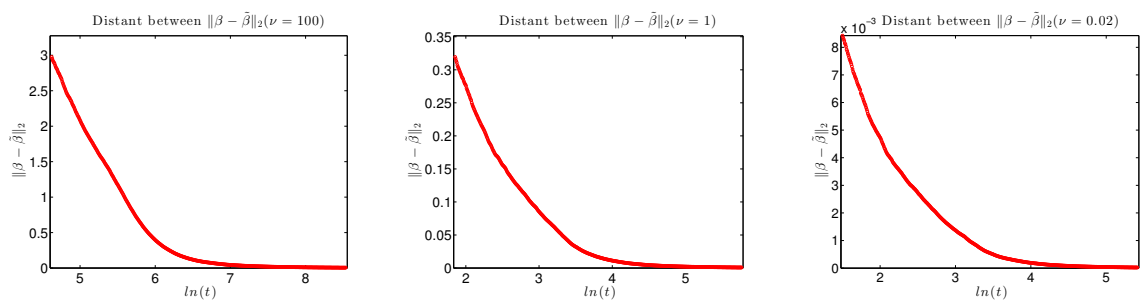

Fig. 6. $\|\beta-\tilde{\beta}\|_{2}$ in the regularized solution path when $\nu=100,1,0.02$. As $\nu$ decreases, the distance of $\beta(t)$ and $\tilde{\beta}(t)$ are tended to be with smaller distance. 

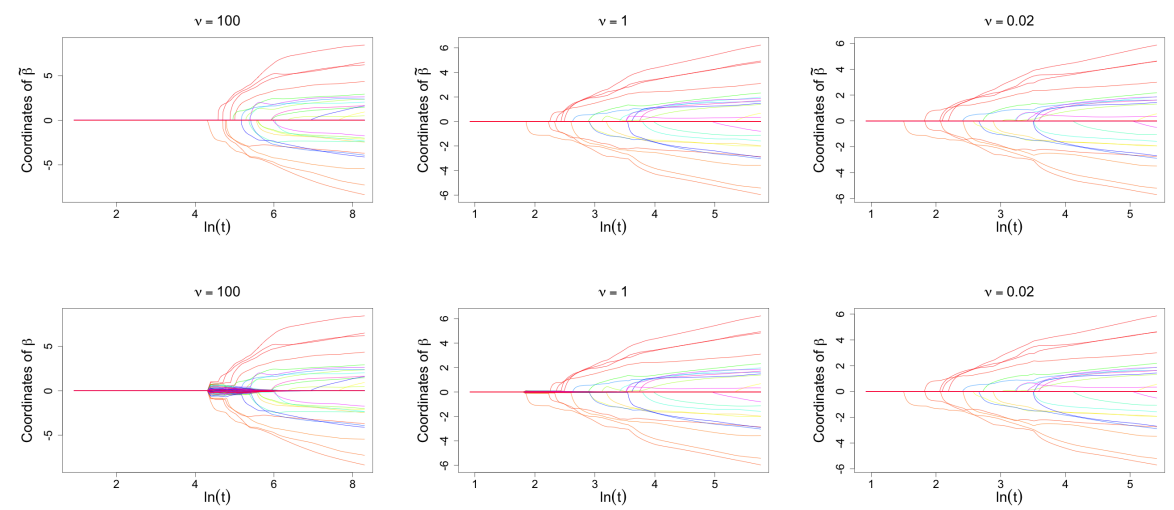

Fig. 7. Comparison of regularized solution path between $\beta$ and $\tilde{\beta}$ when $\nu=100,1,0.02$. They look more similar with each other as $\nu$ decreases.

Since $\|\beta(t)-\tilde{\beta}(t)\|_{2} \rightarrow 0$ as $t \rightarrow+\infty$ and $\tilde{\beta}$ is sparse, it follows that $\beta$ will approximate to $\tilde{\beta}$ on those selected features. In addition to these selected features, before convergence to $\tilde{\beta}, \beta$ can capture other features to better fit data(minimize training error), especially for those ones that significantly correlated with data.

\section{Choice of $\nu$}

The choice of $\nu$ is task-dependent. For stable feature selection, $\nu$ with rather "small" value is suggested. It's noted that $\beta-\tilde{\beta} \rightarrow 0$ as $\nu \rightarrow 0^{+}$, which is reflected by $l_{2}$ norm and regularized solution path shown in figure 6,7 . In this case, the estimator $\tilde{\beta}$ will be constrained in comparably lower dimension space, therefore it may fit data with more stability, notwithstanding $\beta$ have no ability to select other features.

For prediction estimation, the appropriately large value of $\nu$ is preferred. On one hand, when $\nu$ is appropriately large, the ability of selecting features with better model selection consistency can be achieved and $\beta$ will share closer values on these selected features as $t$ progress, as shown in figure 7 . On the other hand, $\beta$ may increase the ability of fitting data by having other features being non-zeros as long as $\nu$ is not too small. In fact, it is shown in table B that comparable results can be given as long as $\nu$ belongs to a reasonable range of values(0.1-10 in this case).

\section{E IDS of ADNI subject used in our experiments}

\begin{tabular}{c|c|c|c|c|c|c}
\hline Subject & ID & Class || Subject $\mid$ ID $\mid$ Class $\mid$ Subject $\mid$ ID $\mid$ Class \\
\hline 123_S_0094 & 9655 & 15AD || 027_S_0408 & 14964 & 15MCI || 072_S_0315 & $12559 \mid 15 \mathrm{NC}$
\end{tabular}




\begin{tabular}{|c|c|c|c|c|c|c|c|c|}
\hline 3_S_0088 & 88 & $A D$ & _S_0481 & $|15044|$ & & | 137_S_0301 & & \\
\hline & 0146 & $\mathrm{AD}$ & 7_S_0417 & & & 002_S_0295 & 13722 & D- \\
\hline & 404 & $\mathrm{AD}$ & 507 & 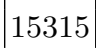 & I & 0327 & 802 & \\
\hline & 962 & $\mathrm{AD}$ & 531 & 431 & MCI & 027_S_0403 & 146 & \\
\hline 0216 & 101 & $\mathrm{AD}$ & 3_S_0567 & 459 & $15 \mathrm{MCI}$ & 137_S_0459 & 4178 & 10 \\
\hline & 203 & $\mathrm{AD}$ & 394 & 510 & [CI & 413 & 437 & NG \\
\hline & 604 & $\mathrm{AD}$ & & & & & & \\
\hline & 327 & D & & 15622 & ACI & & & \\
\hline 316 & 616 & D & 460 & 15711 & ICI & 0488 & 38 & C \\
\hline 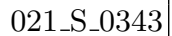 & 12979 & $\mathrm{AD}$ & 542 & & CI & & & NC \\
\hline & 3004 & $\mathrm{AD}$ & & & & & 99 & $\mathrm{NC}$ \\
\hline & 525 & $\mathrm{AD}$ & & & & & & \\
\hline 0370 & 4122 & $\mathrm{AD}$ & 01 & 16 & CI & & 6347 & $\sqrt{ } \mathrm{C}$ \\
\hline 31 & 497 & & 52 & & & & 366 & $\mathrm{NC}$ \\
\hline 54 & 994 & $\mathrm{AD}$ & & & & & 5415 & $\mathrm{NC}$ \\
\hline 28 & 150 & $\mathrm{AD}$ & & 1 & & 36 & 74 & NC \\
\hline & 377 & & & & & & 21 & \\
\hline 9 & 392 & & & & & & 60 & $\mathrm{NC}$ \\
\hline 497 & 6666 & $\mathrm{D}$ & & & & & 48 & $\mathrm{NC}$ \\
\hline & 632 & $\mathrm{AD}$ & & & & & 24 & $\mathrm{CC}$ \\
\hline & 175 & $\mathrm{D}$ & & & & & & \\
\hline & 19585 & & & & & & & $\mathrm{NC}$ \\
\hline 724 & 772 & & & & & & 78 & $\mathrm{NC}$ \\
\hline & 990 & $\mathrm{AD}$ & & & & 12 & 84 & $\mathrm{NC}$ \\
\hline & 169 & & & & & & 03 & \\
\hline 137 & 23112 & & & & & & & \\
\hline 6 & 23231 & & & & & & & \\
\hline 100 & 23581 & $\mathrm{D}$ & & & & & 68 & $15 \mathrm{NC}$ \\
\hline & 3787 & $\mathrm{D}$ & & & & 86 & 13 & $\mathrm{NC}$ \\
\hline & 4863 & $\mathrm{D}$ & & & & 77 & 20 & $\mathrm{NC}$ \\
\hline & 25026 & & & & & & & $\mathrm{NC}$ \\
\hline 126 & 25172 & $\mathrm{AD}$ & 671 & 17 & & 09 & 18589 & $15 \mathrm{NC}$ \\
\hline 0 & 5645 & $\mathrm{AD}$ & & & & & 18896 & $\mathrm{NC}$ \\
\hline 006 & 25816 & $A D$ & 98 & 18 & & 03 & 19155 & $15 \mathrm{NC}$ \\
\hline 002 & 26170 & $\mathrm{AD}$ & & & & & 19258 & $15 \mathrm{NC}$ \\
\hline 130 & 27032 & $\mathrm{AD}$ & 23 & & & & 19567 & $15 \mathrm{NC}$ \\
\hline & 27782 & & & & & 009 & 20013 & $\mathrm{NC}$ \\
\hline & 9381 & $\mathrm{AD}$ & & & & & 20370 & $15 \mathrm{NC}$ \\
\hline & 29579 & $15 \mathrm{AD}$ & & & & & 20543 & $15 \mathrm{NC}$ \\
\hline 029 & 30618 & $\mathrm{AD}$ & 25 & 19 & & 02 ? & 23213 & $15 \mathrm{NC}$ \\
\hline 9 & 31239 & $\mathrm{AD}$ & 669 & & & & 23350 & $15 \mathrm{NC}$ \\
\hline & 252 & & & & & & 23468 & $5 \mathrm{NC}$ \\
\hline & 31576 & & & & & 02 & 24249 & $15 \mathrm{NC}$ \\
\hline 094 & 31678 & $15 \mathrm{AD}$ & 722 & 07 & $\mathrm{CI}$ & 009 & 25128 & $15 \mathrm{NC}$ \\
\hline 109 & 31784 & $15 \mathrm{AD}$ & 709 & & $15 \mathrm{MCI}$ & 09 & 25255 & $15 \mathrm{NC}$ \\
\hline & 5190 & & & & & & 25427 & $\mathrm{NC}$ \\
\hline & 35211 & $15 \mathrm{AD}$ & 558 & 200 & & & 25455 & $5 \mathrm{NC}$ \\
\hline 031_S_1209 & 36178 & $15 \mathrm{AD}$ & 0668 & 20202 & $15 \mathrm{MCI}$ & 006_S_0498 & 25790 & $15 \mathrm{NC}$ \\
\hline & 36269 & $15 \mathrm{AD}$ & 0800 & 20500 & $15 \mathrm{MCI}$ & 052_S_0951 & 26642 & $15 \mathrm{NC}$ \\
\hline 27_S_1081 & 37145 & $15 \mathrm{AD}$ & 002_S_0782 & 20519 & $15 \mathrm{MCI}$ & 130_S_0969 & 26688 & $15 \mathrm{NC}$ \\
\hline
\end{tabular}




\begin{tabular}{|c|c|c|c|c|c|c|c|}
\hline & & & & & & & \\
\hline 4 & 350 & $\mathrm{AD}$ & 752 & $2309715 \mathrm{M}$ & 985 & bur & \\
\hline 254 & 859 & $\mathrm{AD}$ & & & & 1 & \\
\hline & 3395 & & & 1415 & & & \\
\hline & 593 & & & & & & \\
\hline & 617 & & 821 & & & & \\
\hline & 114 & & 771 & & & & \\
\hline & 527 & & & & & & \\
\hline & 344 & & & 1 & & & \\
\hline & 2930 & & & & & & \\
\hline & 060 & & & & & & \\
\hline & 790 & & & & & & \\
\hline & 20 & & & & & & \\
\hline & & & & & & & \\
\hline & 172 & & & & & & \\
\hline & 560 & & & & & & \\
\hline & & & & & & & \\
\hline & & & & & & & \\
\hline & 210 & & & & & & \\
\hline & & & & & & & \\
\hline & 20681 & & & & & & \\
\hline & 24734 & & & & & & \\
\hline & & & & & & & \\
\hline & 29230 & & & & & & \\
\hline & 33832 & & & & & & C \\
\hline & 379 & & & & & & \\
\hline & 46 & & & & & & \\
\hline & & & & & & & \\
\hline & 517 & & & 2 & & & \\
\hline & & & & & & & 30 \\
\hline & & & & & & & \\
\hline & & & & & & & \\
\hline & & & & & & & \\
\hline & & & & 71 & & 49 & $30 \mathrm{NC}$ \\
\hline & & & & & & & \\
\hline & & & & & & & \\
\hline & & & & 29 & & & $30 \mathrm{NC}$ \\
\hline & 267595 & & & 29 & & 61 & $30 \mathrm{NC}$ \\
\hline & & & & 2 & & & \\
\hline & & & & 2 & & & \\
\hline & & & & 2 ? & & & 30 \\
\hline & & & & 30 & & & $30 \mathrm{NC}$ \\
\hline & & & & 30 & & & $30 N$ \\
\hline & & & & & & & \\
\hline & & & & 30 & & & 30 \\
\hline & 292433 & & & 309601 & & 243 & $30 \mathrm{NC}$ \\
\hline & 294209 & & & 31 & & & $30 \mathrm{~N}$ \\
\hline & & & & & & & \\
\hline & & & & 3179915 & & & \\
\hline 130_S_4660 & 300034 & $30 \mathrm{AD}$ & 126_S_1077 & $3185015 \mathrm{~N}$ & 127_S_4148 & 250137 & $30 \mathrm{~N}$ \\
\hline
\end{tabular}


019_S_4549 $300335 \mid$ 30AD || 037_S_0588 $32151 \mid$ 15MCI|| 003_S_4119 |250894|30NC

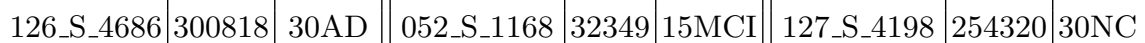

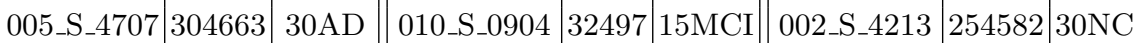
021_S_4718 304749 30AD $\quad$ 002_S_1155 33393 15MCI $\quad$ 031_S_4218 255978 30NC

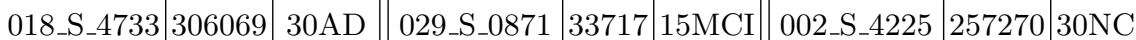

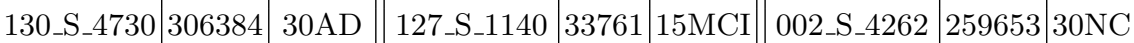
137_S_4756 307118 30AD 027_S_4801 314034 30AD 027_S_4802 317195 30AD 006_S_4867 322012 30AD 016_S_4887 325649 30AD 007_S_4911 328196 30AD 021_S_4924 331257 30AD 137_S_4756 332930 30AD 127_S_4940 335512 30AD 027_S_4938 336926 30AD 027_S_4962 338558 30AD 130_S_4982 341787 30AD 130_S_4984 342274 30AD 130_S_4971 342338 30AD 127_S_4992 342697 30AD 019_S_5012 343916 30AD 019_S_5019 345663 30AD 002_S_5018 346242 30AD 127_S_5028 346696 30AD 130_S_4997 347410 30AD 005_S_5038 351432 30AD 127_S_5056 353203 30AD 127_S_5058 354636 30AD 007_S_0128 10007 15MCI 010_S_0161 10077 15MCI 021_S_0141 10173 15MCI 127_S_0112 10419 15MCI 128_S_0135 10431 15MCI 128_S_0138 10438 15MCI 098_S_0160 $10466 \quad 15 \mathrm{MCI}$ 123_S_0108 $10738 \quad 15 \mathrm{MCI}$ 037_S_0150 10773 15MCI 027_S_0116 10783 15MCI 128_S_0188 10897 15MCI 014_S_0169 10987 15MCI 021_S_0178 10993 15MCI 128_S_0205 11011 15MCI 128_S_0200 11012 15MCI 037_S_0182 11121 15MCI 137_S_0158 11127 15MCI 029_S_0914 33775 15MCI 100_S_1154 $34258 \quad 15 \mathrm{MCI}$ 094_S_1188 34619 15MCI 012_S_1165 35052 15MCI 133_S_0913 35171 15MCI 012_S_1175 $35342 \quad 15 \mathrm{MCI}$ 126_S_1187 36364 15MCI 009_S_1199 36373 15MCI 029_S_1215 37129 15MCI 116_S_0890 37182 15MCI 100_S_1226 $37251 \quad 15 \mathrm{MCI}$ 005_S_1224 $37284 \quad$ 15MCI 037_S_1225 37364 15MCI 029_S_1218 $37373 \quad 15 \mathrm{MCI}$ 027_S_1213 $37393 \quad 15 \mathrm{MCI}$ 127_S_1210 38319 15MCI 116_S_1243 38462 15MCI 033_S_1309 38837 15MCI 027_S_1277 39715 15MCI 129_S_1246 40237 15MCI 129_S_1204 $40398 \quad 15 \mathrm{MCI}$ 033_S_1284 40881 15MCI 033_S_1279 40902 15MCI 029_S_1318 41062 15MCI \begin{tabular}{l|l|l|} 
116_S_1271 & 41321 & $15 \mathrm{MCI}$
\end{tabular} 094_S_1330 $41491 \quad 15 \mathrm{MCI}$ 121_S_1322 $42188 \quad 15 \mathrm{MCI}$ 094_S_1314 42694 15MCI 052 _S_1352 42876 15MCI 123_S_1300 43214 15MCI 121_S_1350 44122 15MCI 072_S_1211 44137 15MCI 116_S_1315 44143 15MCI 052_S_1346 $44515 \quad 15 \mathrm{MCI}$ 027_S_1387 44748 15MCI 024_S_1393 44887 15MCI 132_S_0987 $45815 \quad 15 \mathrm{MCI}$ 029_S_1384 47455 15MCI 072_S_1380 49799 15MCI 094_S_1398 $53551 \quad 15 \mathrm{MCI}$ 128_S_0225 11179 15MCI $\quad$ 024_S_1400 53739 15MCI 136_S_0107 11227 15MCI 094 _S_1417 $60175 \quad$ 15MCI 032_S_0214 $11280 \quad 15 \mathrm{MCI} \quad 127$ _S_1419 61670 15MCI 941_S_4100 $25978130 \mathrm{NC}$ 002_S_4264 259796 30NC 021_S_4276 260047 30NC 029_S_4290 260425 30NC 098_S_4275 $26145930 \mathrm{NC}$ 094_S_4234 261531 30NC 018_S_4257 262076 30NC 136_S_4269 264215 30NC 029_S_4279 265980 $30 \mathrm{NC}$ 021_S_4335 266174 30NC 130_S_4343 266217 30NC 018_S_4349 266625 30NC 129_S_4369 267405 30NC 130_S_4352 267711 30NC 129_S_4371 268462 30NC 018_S_4313 268930 30NC 019_S_4367 269273 30NC 007_S_4387 269929 30NC 036_S_4389 270462 30NC 003_S_4350 270999 30NC 129_S_4422 272184 30NC 018_S_4399 272231 30NC 018_S_4400 273504 30NC 021_S_4421 273564 30NC 029_S_4383 273993 30NC 003_S_4441 277108 30NC 136_S_4433 278511 $30 \mathrm{NC}$ 006_S_4449 279470 30NC 031_S_4474 280369 30NC 007_S_4488 281560 30NC 006_S_4485 281882 30NC 010_S_4345 282005 30NC 031_S_4496 282638 30NC 098_S_4506 282934 30NC 094_S_4459 283445 30NC 094_S_4460 283573 30NC 010_S_4442 283915 30NC 007_S_4516 284424 30NC 029_S_4385 285589 30NC 094_S_4503 286222 30NC 073_S_4559 $286553 \quad 30 \mathrm{NC}$ 021_S_4558 $287527 \quad 30 \mathrm{NC}$ 109_S_4499 288999 30NC 005_S_0222 11299 15MCI $\mid$ 137_S_1414 $64472 \mid$ 15MCI $\mid$ 100_S_4469 $289564 \mid$ 30NC 


\begin{tabular}{|c|c|c|c|c|c|c|c|c|}
\hline 7_S_0179 & 11348 & FVI & |127_S_1427 & $\mathrm{I}^{-1}$ & $15 \mathrm{M}$ & |100_S_4511 & 2896 & 800 \\
\hline 11_S_0231 & 11430 & $15 \mathrm{MCI}$ & 037_S_1421 & 70885 & $15 \mathrm{MCI}$ & 012_S_4545 & 290413 & $30 \mathrm{~N}$ \\
\hline 7_S_0249 & 11544 & $15 \mathrm{MCI}$ & 137_S_1426 & 72082 & $15 \mathrm{MCI}$ & 053_S_4578 & & $0 \mathrm{NC}$ \\
\hline 0269 & 11615 & MCI & 007_S_0041 & 8177 & $15 \mathrm{MCI}$ & 127_S_4604 & & $\mathrm{NC}$ \\
\hline 0_S_0289 & 11850 & $5 \mathrm{MCI}$ & 123_S_0050 & 8648 & $15 \mathrm{MCI}$ & 007_S_4620 & 2939 & $\mathrm{IN}$ \\
\hline 1_S_0273 & 11942 & $5 \mathrm{MCI}$ & 100_S_0006 & 8793 & $15 \mathrm{MCI}$ & 127_S_4645 & 295590 & $30 \mathrm{NC}$ \\
\hline _S_0293 & 11982 & $5 \mathrm{MCI}$ & 007_S_0101 & 9602 & $15 \mathrm{MCI}$ & 002_S_4270 & 260581 & $\mathrm{ONC}$ \\
\hline S_0294 & 12065 & $5 \mathrm{MCI}$ & 123_S_0106 & 10126 & $15 \mathrm{NC}$ & 013_ & 296776 & $\mathrm{~N}$ \\
\hline _S_0276 & 12092 & $5 \mathrm{MCI}$ & 100_S_0035 & 8120 & $15 \mathrm{NC}$ & -4580 & 296859 & $30 \mathrm{~N}$ \\
\hline 8_S_0227 & 12119 & $\mathrm{MCI}$ & 100_S_0047 & 8899 & $15 \mathrm{NC}$ & _4642 & 296878 & $30 \mathrm{NC}$ \\
\hline 7_S_0256 & 12250 & $5 \mathrm{MCI}$ & 010_S_0067 & 9093 & $15 \mathrm{NC}$ & 01 & 29 & $\mathrm{NC}$ \\
\hline S_0285 & 12424 & $\mathrm{CI}$ & 018_S_0043 & 9324 & $15 \mathrm{NC}$ & 029 & 29 & $\mathrm{NC}$ \\
\hline 0288 & 12654 & $\mathrm{ICI}$ & 100_S_0069 & 9417 & $15 \mathrm{NC}$ & 013 & & $\mathrm{NC}$ \\
\hline 0344 & 12697 & $15 \mathrm{MCI}$ & 032 & 9680 & $15 \mathrm{NC}$ & 02 ? & & $30 \mathrm{NC}$ \\
\hline 1_S_0332 & 12862 & $5 \mathrm{MCI}$ & 123_S_0072 & 9752 & $15 \mathrm{NC}$ & 4632 & 30 & $30 \mathrm{NC}$ \\
\hline 258 & 13085 & $5 \mathrm{MCI}$ & 007 & 10027 & $15 \mathrm{NC}$ & 094 & 30 & $\mathrm{NC}$ \\
\hline 0307 & 13281 & MCI & $131_{-}$ & 10043 & $15 \mathrm{NC}$ & 4638 & 30 & $30 \mathrm{NC}$ \\
\hline 3_S_0390 & 13315 & $5 \mathrm{MCI}$ & 027_S_0118 & 11370 & $15 \mathrm{NC}$ & $01:$ & & $30 \mathrm{NC}$ \\
\hline 1_S_0351 & 13783 & $15 \mathrm{MCI}$ & 098_S_0172 & 11398 & $15 \mathrm{NC}$ & 136_S_4726 & 308 & $30 \mathrm{NC}$ \\
\hline S_0424 & 13909 & CI & 130_S_0232 & 11567 & $15 \mathrm{NC}$ & -4688 & 31 & $30 \mathrm{NC}$ \\
\hline S_0389 & 13938 & $15 \mathrm{MCI}$ & 005_S & 11645 & $15 \mathrm{NC}$ & 019 & 315857 & $30 \mathrm{NC}$ \\
\hline 94_S_0434 & 13964 & $15 \mathrm{MCI}$ & 123_S_0113 & 11714 & $15 \mathrm{NC}$ & 127_S_4843 & 316771 & $30 \mathrm{NC}$ \\
\hline 8_S_0401 & 14161 & $15 \mathrm{MCI}$ & 128_S_0230 & 11806 & $15 \mathrm{NC}$ & 003 & 319414 & $30 \mathrm{NC}$ \\
\hline _0409 & 14240 & & 137 א_137 & 12028 & $15 \mathrm{NC}$ & 00 & & $30 \mathrm{~N}$ \\
\hline 16_S_0361 & 14296 & $15 \mathrm{MCI}$ & 128_S_0245 & 12242 & $15 \mathrm{NC}$ & 003_S_4872 & 321376 & $30 \mathrm{NC}$ \\
\hline 132_S_0339 & 14367 & $15 \mathrm{MCI}$ & 128_S_0272 & 12313 & $15 \mathrm{NC}$ & 003_S_4900 & 325729 & $30 \mathrm{NC}$ \\
\hline _S_0377 & 14405 & $15 \mathrm{MCI}$ & 128_S_0229 & 12459 & $15 \mathrm{NC}$ & 016_S_4951 & 337692 & $30 \mathrm{NC}$ \\
\hline 0485 & 14928 & & 021_ & 12466 & $15 I$ & & & \\
\hline _S_0102 & 9709 & & 098_S_0171 & 10818 & $15 \mathrm{NC}$ & & & \\
\hline
\end{tabular}

